\title{
Tactical Population Movements and Distributions for Ideally Motivated Competitors
}

\author{
Jonathan T. Rowell \\ University of North Carolina, Chapel Hill, North Carolina 27599 \\ Submitted December 4, 2009; Accepted July 24, 2010; Electronically published September 21, 2010 \\ Online enhancements: appendixes.
}

Department of Ecology and Evolutionary Biology, University of Tennessee, Knoxville, Tennessee 37996; and Department of Biology,

ABstract: The spatial distributions of populations are a reflection of underlying rules for movement behavior in the context of the environment encountered by individuals. Here I study how ideal directed movement - in which individuals travel in the direction offering the most immediate perceived improvement to their personal fitness-dictates the spatial position of two populations occupying the same relative niche and engaged in competition via interference to an individual's ability to gather resources. Drawing on the analytic derivation of equilibria, numerical simulations, and graphical assessments, I provide conditions under which sympatry, parapatry, or regional exclusion is expected during different phases of the community's development. I also demonstrate that specific competitive asymmetries produce distinguishable distributions and invasion patterns and identify which populations are found centrally or peripherally. Dynamic and dispersal equilibria were examined for differences in the sensitivity to spatial variations in fitness, per capita mortality, metabolic efficiency, the strength of interspecific interference, resource collection speed, and the optimal location of each population along an environmental cline. These asymmetries were studied both in isolation and pairwise in fitness trade-off scenarios.

Keywords: ideal directed movement, sympatry, density-dependent fitness, interference competition, invasion, fitness trade-off.

The development of a mechanistic framework describing how individual objectives and behavioral responses ultimately translate into distributions and range shifts at the population level remains an open problem in ecology (Lima and Zollner 1996; Gaston 2003; Moorcroft and Lewis 2006). Numerous biotic and abiotic factors influence population distributions (Holt and Keitt 2005; Goldberg and Lande 2007) via local dynamics and also through habitat selection, whether the latter process is simple chemotaxis displayed by bacteria or slime molds (Berg 1993) or preytaxis (Kareiva and Odell 1987) or infotaxis (Ver-

* E-mail: jtrowell@email.unc.edu.

Am. Nat. 2010. Vol. 176, pp. 638-650. (C) 2010 by The University of Chicago. 0003-0147/2010/17605-51775\$15.00. All rights reserved.

DOI: $10.1086 / 656494$ gassola et al. 2007) among higher organisms. Even cultural measures, for example, similarity in song repertoires among passerine birds (Podos and Warren 2007), can guide distribution establishment. Competitive pressures may be the primary determinant of range limits among these factors (Gaston 2003; Case et al. 2005; Moorcroft and Lewis 2006; Krrivan et al. 2008). Both conspecific (Fretwell and Lucas 1969; Rosenzweig 1991; Fryxell et al. 2004; Hancock and Milner-Gulland 2006) and heterospecific (Kirkpatrick and Barton 1997; Peterson 2003) competitors within the larger community assemblage affect an individual's fitness. The literature exploring direct or indirect competition at the local dynamics level is extensive, but habitat selection theory for multiple distinct populations is still incomplete (Křivan et al. 2008), particularly for systems presented within a structured, spatially explicit context. This article contributes to the development of this larger theory by studying the transient and steady state distribution patterns of two interfering populations whose individuals advance along personal fitness gradients within a heterogeneous, continuous landscape and between whom exist one or more competitive asymmetries.

Habitat selection strategies are expressed as part of the populations' dispersal dynamics, which subsequently influence individual and population fitness levels. The measure of fitness within a habitat-or observable correlated proxies such as temperature, resource abundance, crowdedness, or salinity levels-also potentially serves a dual function as a public cue by which responsive organisms can further adjust their position within the larger environment. Cue assessment can take many forms and even occur independent of any direct interaction between antagonists. Examples include the visual inspection of per capita resource abundance among patches (Fretwell and Lucas 1969), granivores judging the distance from seed sources (Mari et al. 2008), and olfactory detection of scent marking by wolves (Moorcroft and Lewis 2006) and turtles (Polo-Cavia et al. 2009). The habitat strategies employed 
then are not fixed but continuously updated to reflect feedback from current decisions.

The literature has employed a confusing variety of terms for such processes (see Edelaar et al. 2008), so in an effort to provide clarification for this article, I use the term "directed movement" for the broader class of active, objective-oriented movement predicated on the current state of the environment at different scales that cannot be ascribed to mere randomness (e.g., diffusion) or external transport (e.g., convection). This category encompasses chemotaxis and similar processes controlled by environmental signals. I retain "better response dynamics" for that subset of relocations made in pursuit of areas granting higher individual fitness (Křivan et al. 2008; sensu Hofbauer and Sigmund 1998). Matching habitat choice (Ravigné et al. 2004), fitness gradient climbing (Shigesada et al. 1979), and the classic ideal free distribution (IFD; Fretwell and Lucas 1969) are all examples of better response dynamics. Following Rowell (2009) I further classify better response dynamics as "ideal directed movement" (or simply "ideal") when it operates under the principles of a locally restricted ideal free domain for some density-dependent fitness function or search measure over a spatially explicit landscape. Thus, an individual's ability to evaluate the landscape for resources and competition is limited to the most immediate gradient scale or neighborhood. Like the classic IFD formulation, there is no cost to moving, nor is there any inherent advantage granted to residents over newcomers. Individuals or populations characterized by this form of movement are said to be "ideally motivated."

In this article, I show how the response of ideally motivated individuals to competition within and between groups organizes the development of population distributions within a heterogeneous resource landscape. This article builds directly on the continuous model of a single ideally motivated population (Rowell 2009; see also Kshatriya and Cosner 2002; Cosner 2005) by incorporating a second population with which the first engages in interference competition for a common resource (Wilson 1980; López-Gómez and Molina-Meyer 2006). The underlying theoretical framework synthesizes a number of recent modeling developments related to better response dynamics (Grindrod 1988; Armsworth and Roughgarden 2005; Cosner 2005; Cressman and Krrivan 2006), and its techniques and conclusions are broadly applicable to other competition or community models. Population densities are described by a pair of reaction-advection equations (Shigesada et al. 1979), where the rates of movement are proportional to the gradients of density-dependent fitness measures. As interspecific competition is often not symmetric (Schoener 1983), I build into the model several potential interpretations of competitive asymmetry that can be analyzed separately or in conjunction with one another. Those differences studied include sensitivity to the fitness gradient, metabolic efficiency and mortality, interference dominance, resource collection, and the populations' respective optimal locations along an environmental cline. I show that different asymmetries lead to the adoption of distinct, recognizable spatial tendencies during both the near-term (transient or invasive) and the longterm (steady state) phases of the community, and I also demonstrate whether a given population's primary habitat is centrally or peripherally located. I provide conditions under which ideal populations overlap sympatrically, when they must abut one another parapatrically, and when regional exclusion due to a competitive advantage is or is not avoidable.

\section{Model of Two Competing Species with Ideal Movement}

This section presents a dispersal-competition model for two ideally motivated populations. It then formally defines the continuous IFD and describes analytic and graphical techniques that assess whether local or regional co-occurrence of two ideal populations is possible. Finally, I provide resource levels corresponding to changes in co-occurrence results.

Consider two regionally co-occurring, ideally motivated populations $(i=1,2)$ with densities $u_{1}(x, t)$ and $u_{2}(x, t)$ at position $x$ and time $t$. The populations compete via density-dependent interference for a common standing resource whose abundance or quality is described by a unimodal curve, $R(x)$, that approaches 0 at the physical limits of the environment and is constant over time $(\partial R / \partial t=$ 0 , e.g., quasi-steady state assumption with rapid replacement). The per capita rate at which individuals recover resources is given by the local ratio of available resources, $R$, to the time spent either in competition or in basic search or retrieval activities:

$$
S_{i}\left(R, u_{1}, u_{2}\right)=\frac{R}{a_{i 1} u_{1}+a_{i 2} u_{2}+h_{i}} .
$$

The parameter $h_{i}$ represents an intrinsic per-area harvest time, while $a_{i j}$ is the competition pressure that a member of population $i$ feels in the presence of a member of population $j$. Territoriality, aggression, relative fighting strength, and degree of overlap in resource needs all contribute to these interference terms. Where specificity is required, I consider the following three primary scenarios: (1) both populations are equally capable of competing over resources $\left(a_{i j}=1\right)$, (2) interspecific pressures are equal but distinct from intraspecific ones $\left(a_{12}=a_{21}=a \neq 1\right)$, and (3) the first population competitively dominates the second $\left(a_{12}=1-\epsilon, a_{21}=1+\epsilon\right)$. In all three cases, within-group pressures are normalized $\left(a_{i i}=1\right)$ to pro- 
vide a common baseline for comparison. Other measures (e.g., mass-action, per-item handling, or Allee effect models) are also possible forms of $S_{i}$ (Rowell 2009), but in conjunction with equation (2), this particular expression presents a reasonable balance between the number of contrastable features and tractability.

The term $S_{i}$ measures only one component of fitness (resource accrual rate), but it is an honest public signal of the state of the environment. Individuals perceive local variations in $S_{i}$ with sensitivity $k_{i}$ and travel up its gradient in the direction of greatest immediate increase. At the population level, locally ideal movement yields a directional flow, $J_{i}=k_{i} u_{i}\left(\partial S_{i} / \partial x\right)$. Populations convert accrued resources into per capita growth with metabolic efficiency, $r_{i}$, and suffer a uniform mortality rate, $\mu_{i}$. The resulting dispersal-competition model is a pair of partial differential equations $(i=1,2)$ :

$$
\frac{\partial u_{i}}{\partial t}=-k_{i} \frac{\partial}{\partial x}\left(u_{i} \frac{\partial S_{i}}{\partial x}\right)+r_{i} u_{i} S_{i}-\mu_{i} u_{i} .
$$

This system is closed, with no migration across its boundaries.

\section{Continuous IFDs and Personal Fitness Landscapes}

For the purposes of discussing ideal distributions, it is assumed that equation (2) is a "rapid" model (Křivan and Sirot 2002; Abrams et al. 2007), in which dispersal dynamics operate on a faster timescale than do local population dynamics. A population is said to be in a continuous IFD (sensu Rowell 2009; see also the pioneering work of Kshatriya and Cosner 2002; Cosner 2005) for its current overall size if $S_{i}$ is (1) uniformly equal to a common value, $S_{i}=C_{i}$, over a contiguous area where its density is nonzero and (2) less than $C_{i}$ within some neighborhood of that area. This definition holds whether a population exists in isolation or has a competitor.

Under the rapid-model assumption, Rowell (2009) previously showed that the density of a single transient population under normalized pressure $\left(u_{2} \equiv 0, a_{11}=1\right)$ converged toward a continuous IFD (fig. $1 A$ ):

$$
\tilde{u}_{1}= \begin{cases}1 / a_{11}\left[\left(R / C_{1}\right)-h_{1}\right], & R(x) \geq C_{1} h_{1} \\ 0, & \text { otherwise }\end{cases}
$$

Moreover, there is a one-to-one inverse relationship between $C_{i}$ and the regional population size (fig. $1 B$ ), provided the inhabited region is connected. Geographically distinct subpopulations-perhaps generated by multiple resource peaks or competition-driven fragmentation-adhere to local constraining values, not global ones. The unique steady state attractor characterized by the population's effective mortality (the ratio of per capita mortality to metabolic conversion efficiency, $C_{\mathrm{le}}=\mu_{1} / r_{1}$ ), is both a dispersal equilibrium and a demographic equilibrium.

For nonideal populations, the plot of $S_{i}(x)$ graphically represents the collective population's habitat viability or preference views. Areas of greater or lesser appeal are revealed, respectively, as peaks and valleys on this personal fitness landscape (fig. $1 \mathrm{~A}, 1 \mathrm{C}$ ), while the slope of the landscape is proportional to the direction and speed of local movement. In this context, continuous IFDs (eq. [3]) correspond to fitness landscapes where $S_{i}$ is uniform (flat) within the inhabited region and inferior in bordering areas (fig. 1A).

\section{Analytic and Graphical Conditions for Ideal Sympatry and Local Exclusion}

In parallel to a single population, two competing populations are each ideal if they are defined by distinct values $S_{i}=C_{i}$, although the distributions will reflect the effects of interspecific competition where they overlap. The value pair $\left(C_{1}, C_{2}\right)$ is often uniquely determined by the populations' regionwide abundances but not necessarily so. Values must remain constant over contiguous subpopulations of a given species, but this is not a global constraint across a collection of locally ideal but disconnected groups, for example, if movement barriers partition a population.

Two ideally distributed populations competing under equations (1) and (2) may be locally sympatric if

$$
\frac{a_{21}}{a_{11}}<\left[\frac{\left(R / C_{2}\right)-h_{2}}{\left(R / C_{1}\right)-h_{1}}\right]<\frac{a_{22}}{a_{12}} .
$$

Persistent sympatry is tested with the populations' effective mortalities, $C_{i \mathrm{e}}=\mu_{i} / r_{i}$. Condition (4) is a behavioral consequent of ideal movement, and it is based on motivational invasibility criteria (app. A in the online edition of the American Naturalist). The left inequality is a necessary condition for the second population to invade the first by immigration, and the right inequality does likewise for the first population. The sympatry condition is spatially dependent by virtue of resource heterogeneity, and the pair $\left(C_{1}, C_{2}\right)$ may permit sympatry in some areas and require exclusion in others.

Satisfaction of only one inequality in condition (4) reveals an area vulnerable to competitive exclusion by displacement, while a complete reversal of the order of inequalities indicates mutual exclusion in which coexistence states are unstable with respect to spatial variations due to high competition (cf. Křivan and Sirot 2002). Areas characterized as such would appear patchy or in a mosaic 

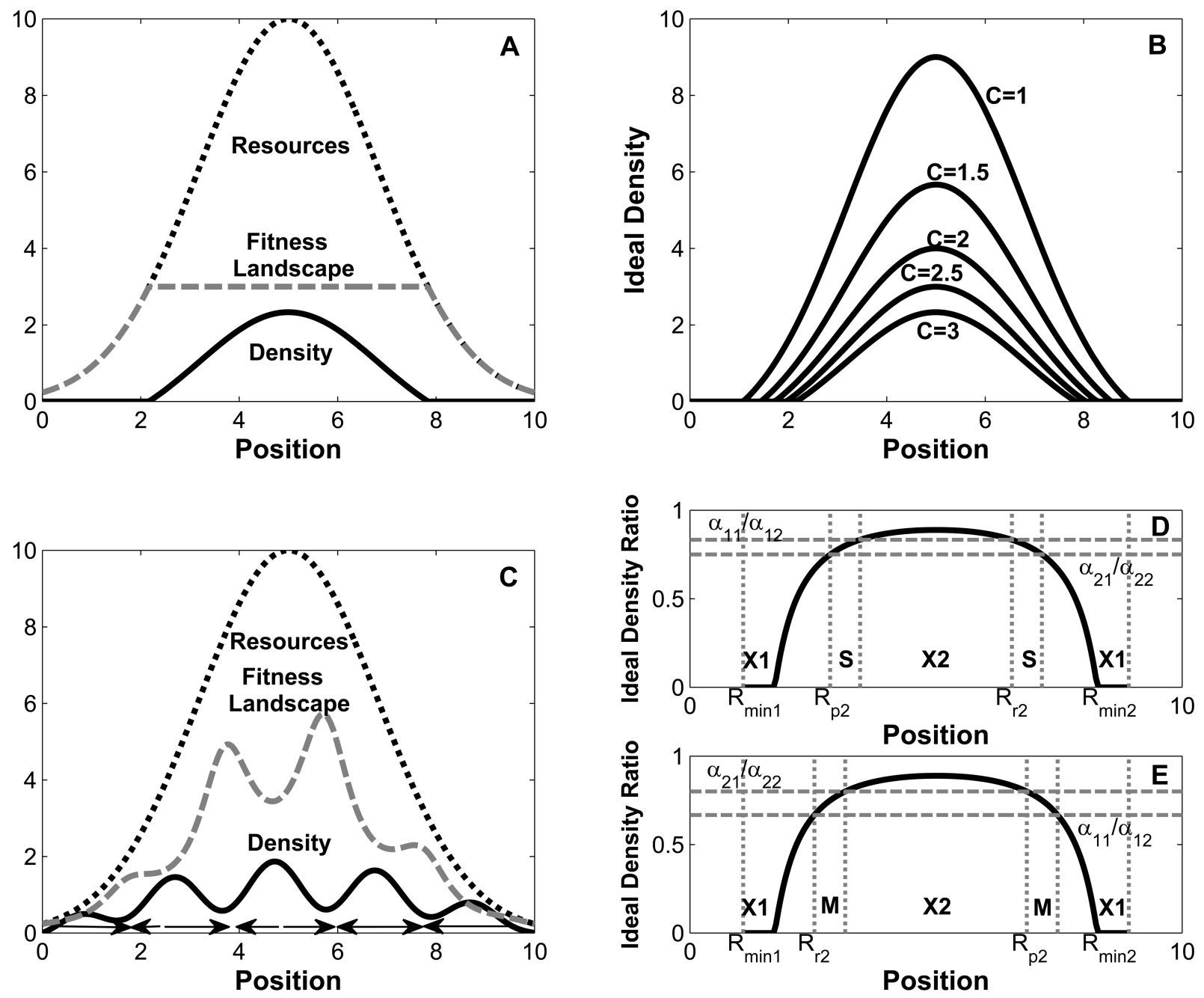

Figure 1: Density, fitness, and competition pressures. The resource curve for all panels (dots [where shown]) is $R(x)=10 \exp \left[-0.15(x-5)^{2}\right]$. Unless stated otherwise, $a_{i j}=h_{i}=1$. A, A single ideally distributed population $(C=3)$ has a maximal uniform fitness $($ dashed lines; $S=C)$ in the populated area. $B$, Different ideal distributions correspond to distinct contour values $(C)$. $C$, A nonideal population produces nonuniform fitness. Arrows indicate current movement. $D$, E, Ideal density ratio condition, $\left(a_{21} / a_{22}\right)<\tilde{u}_{2} / \tilde{u}_{1}<\left(a_{11} / a_{12}\right)$, (equivalent to eq. [4]). Populations may be excluding $(X)$ or sympatric $(S)$ or in a fragmented mosaic $(M)$. Parameters: $C_{1}=C_{2}=2, h_{1}=0.5, h_{2}=1$. Interspecific pressure coefficients: $D$, $\left(a_{12}, a_{21}\right)=(1.2,0.75) ; E,\left(a_{12}, a_{21}\right)=(1.5,0.8)$. Locations noted for minimum $\left(R_{\min }\right)$ and threshold $\left(R_{\mathrm{r}}, R_{\mathrm{p}}\right)$ resources (symmetrically arranged).

pattern reflecting historic movements (fig. 4C, $4 D$ ). Moreover, by itself condition (4) is insufficient to conclude that sympatry or exclusion has actually occurred. There must also have existed an initial distribution and migratory pathway that gave the indicated population access to the subregion before attaining an ideal distribution. Areas positively identified by condition (4) may still lack that population due to movement barriers in adjacent regions (e.g., fig. $2 E, 2 H)$. Figure $1 D, 1 E$ provides a parallel graphical analysis of ideal populations based on the equivalent condition $\left(a_{21} / a_{22}\right)<\left(\tilde{u}_{2} / \tilde{u}_{1}\right)<\left(a_{11} / a_{12}\right)$, where $\tilde{u}_{i}$ is the single- species distribution given by equation (3). Finally, complete equality in the sympatry condition indicates spatially neutral distributions (e.g., fig. $2 A$ ).

\section{Transitional Resource Levels}

In addition to the minimum resource requirements of each population $\left(R=C_{i e} h_{i}\right)$, there are potentially two other critical resource values corresponding to transitions between the predictions of the sympatry condition at population equilibrium (eq. [4], $C_{i \mathrm{e}}$ ). These thresholds indicate 

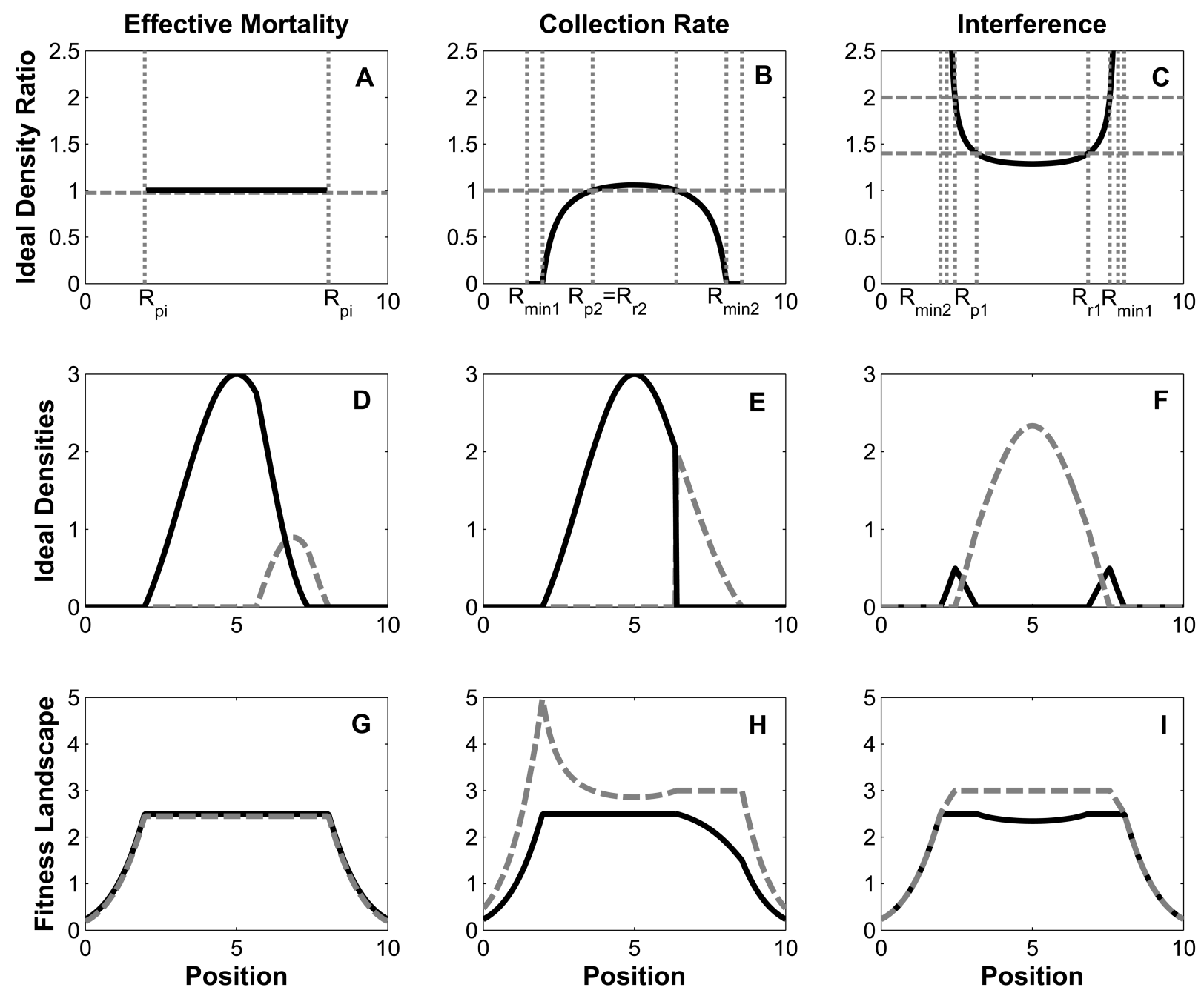

Figure 2: Transient ideal competitors. Two ideal populations are distinguished by a single parameter. Solid lines represent the resident inferior population (population 2). The superior invader (population 1; dashed lines) is assumed to have been introduced from the right. Unless stated otherwise, $C_{1}=3, C_{2}=2.5, a_{i j}=1, h_{i}=1$. Resources are as in figure 1. Parametric contrasts are arranged by column: effective mortality (left; $\left.C_{1}=2.5\right)$, resource gathering (center; $\left.h_{1}=0.5\right)$, and interference strength $\left(\right.$ right $\left.; a_{21}=1.4, a_{12}=0.5\right)$. Plots show ideal density ratios, $\tilde{u}_{2} / \tilde{u}_{1}$ (equivalent to eq. [4]; top row), conforming ideal distributions (middle), and the resulting fitness landscapes (bottom). Effective mortality: distributions are arbitrarily interchangeable as sympatry is possible but not obligatory $(A, D)$, and populations see identical fitness landscapes $(G)$. Resource gathering: parapatry is obligatory $(B)$, but resident population may be a barrier to movement toward preferred locations $(E, H)$. Interference: sympatry at intermediate resources is necessary $(C)$, with strong individuals favoring the central area and weaker fighters staying at the margins $(F, I)$.

where one population may successfully invade or prohibit the invasion of the other population under both ideal movement and local dynamics (derived in app. B in the online edition of the American Naturalist).

If $\left(C_{1 \mathrm{e}} / C_{2 \mathrm{e}}\right)>\left(a_{21} / a_{11}\right)$, we can define the persistence threshold of population $2, R_{\mathrm{p} 2}$, as a minimum resource level above which it can dynamically maintain itself on introduction without regard to the presence of population 1. This persistence threshold is

$$
R_{\mathrm{p} 2}=\frac{C_{1 \mathrm{e}} C_{2 \mathrm{e}}\left(a_{11} h_{2}-a_{21} h_{1}\right)}{a_{11} C_{1 \mathrm{e}}-a_{21} C_{2 \mathrm{e}}}=\frac{\mu_{1} \mu_{2}\left(a_{11} h_{2}-a_{21} h_{1}\right)}{a_{11} \mu_{1} r_{2}-a_{21} \mu_{2} r_{1}} .
$$

Below that threshold, population 2 fails to persist if population 1 is already ideally distributed at population equilibrium. If instead $\left(C_{1 \mathrm{e}} / C_{2 \mathrm{e}}\right)<\left(a_{21} / a_{11}\right)$, then the calculated resource value is a maximum, not a minimum. The nomenclature is changed to reflect that it is the existing res- 
ident that outperforms at higher resources, with the resource value identified as the exclusion threshold for population $1, R_{\mathrm{r} 1}$. The exclusion threshold for population 2 is

$$
R_{\mathrm{r} 2}=\frac{C_{1 \mathrm{e}} C_{2 \mathrm{e}}\left(a_{12} h_{2}-a_{22} h_{1}\right)}{a_{12} C_{1 \mathrm{e}}-a_{22} C_{2 \mathrm{e}}}=\frac{\mu_{1} \mu_{2}\left(a_{12} h_{2}-a_{22} h_{1}\right)}{a_{12} \mu_{1} r_{2}-a_{22} \mu_{2} r_{1}},
$$

provided that $C_{1 \mathrm{e}} / C_{2 \mathrm{e}}>a_{22} / a_{12}$; otherwise, the calculated value is the persistence threshold for population $1, R_{\mathrm{p} 1}$. Strict parapatry occurs when these thresholds coincide.

\section{Results}

Here I examine parametric asymmetries for sensitivity $\left(k_{i}\right)$, effective mortality $\left(\mu_{i} / r_{i}\right)$, interspecific interference $\left(a_{i}\right)$, and resource gathering $\left(h_{i}\right)$, along with a fifth contrast made in the populations' optimal locations along an environmental cline. Table 1 summarizes results for all basic comparisons, and figure 2 illustrates representative ideal transient distributions for those simple asymmetries typically leading to regional exclusion. Figure 3 contains the secondary findings when the five contrasts are combined pairwise as ability trade-offs.

\section{Sensitivity and Effective Mortality}

Neither sensitivity $\left(k_{i}\right)$ nor effective mortality $\left(\mu_{i} / r_{i}\right)$ directly alter the shape of the personal fitness landscape, $S_{i}$. Both populations recognize the same local fitness peaks and valleys when intra- and interspecific interferences are normalized $\left(a_{i j}=1\right)$, regardless of the specific asymmetry (e.g., fig. $2 G$ ), and the community satisfies a common ideal distribution (cf. eq. [3]):

$$
u_{1}+u_{2}= \begin{cases}(R / C)-h, & R \geq C h \\ 0, & \text { otherwise. }\end{cases}
$$

Beyond this joint constraint, however, there is no local implication for the relative frequencies of the two populations. Distributions will retain echoes of historic competition ("ghosts of competition past"; Connell 1980; Rosenzweig 1991). Thus, a more responsive population $\left(k_{i}\right)$ often exhibits a wider overall range because differential movement rates lead to an increased likelihood in colonizing areas, but without a commensurate distinction in fitness postarrival, local coexistence is per force reached (eq. [4] collapses to tautological equality). The community converges to that distribution defined by the common effective mortality, $C_{\mathrm{e}}=\mu / r$.

When competitors differ only in their mortality rates $\left(\mu_{i}\right)$ or metabolic efficiencies $\left(r_{i}\right)$, there is an absence of differential movement rates. Migration alone does not introduce novel points of segregation because co-occurring populations disperse in tandem. Although the two groups do share a common transient ideal distribution (eq. [7]; fig. 2A, 2G), they cannot permanently coexist (cf. parallel nullclines in an analogous spatially homogeneous model). The population with the smaller effective mortality, $\mu_{i} / r_{i}$, ultimately outcompetes its less hale rival by inducing regionwide overcrowding and gradually displacing the less fit population (fig. 2D).

Distinct but symmetric interspecific competition $\left(a_{12}=a_{21}=a \neq 1\right)$ relaxes the requirement that $C_{1}=$ $C_{2}$ during the ideal transient phase. For models characterized more by intraspecific competition $\left(a<1, a_{i i}=1\right)$, the sympatry condition (4) is satisfied for all resource values $R>\max \left\{R_{\mathrm{p} 1}^{t}, R_{\mathrm{p} 2}^{t}\right\}$ (transient analogs to $R_{\mathrm{p} i}$ ), with the numerically larger population exclusively holding the

\begin{tabular}{|c|c|c|c|c|}
\hline Contrasted feature & Transient phase & Steady state phase & $R_{\mathrm{p} 2}$ & $R_{\mathrm{r} 2}$ \\
\hline Sensitivity & Arbitrary & Arbitrary & $\frac{\mu h}{r}$ & \\
\hline Effective mortality & Arbitrary & Exclusion & $\frac{(1-a) \mu_{1} \mu_{2} h}{\mu_{1} r_{2}-\mu_{2} r_{1} a}$ & $\frac{(a-1) \mu_{1} \mu_{2} h}{a \mu_{1} r_{2}-\mu_{2} r_{1}}$ \\
\hline Interference & Overlap & Exclusion & $\frac{\mu h_{\mathrm{a}}}{r}$ & NA \\
\hline Resource collection & Parapatry & Exclusion & $\frac{\mu\left(h_{2}-a h_{1}\right)}{r(1-a)}$ & $\frac{\mu\left(a h_{2}-h_{1}\right)}{r(a-1)}$ \\
\hline Optimal location & Parapatry & Parapatry & $\ldots^{\mathrm{b}}$ & $\ldots^{\mathrm{b}}$ \\
\hline \multicolumn{5}{|c|}{$\begin{array}{l}\text { Note: This table lists the expected transient and steady state spatial arrangements for individual } \\
\text { asymmetries, along with the persistence }(a<1) \text { and exclusion }(a>1) \text { thresholds for the inferior pop- } \\
\text { ulation (here population } 2) \text { under secondary variation in interference. Arbitrary }=\text { an infinite number } \\
\text { of local combinations are possible subject to total population levels; exclusion = the inferior population } \\
\text { is eliminated regionwide; overlap }=\text { both populations are present, with an intermediate zone of sym- } \\
\text { patry; parapatry = populations exist in distinct ranges. } \\
\text { a Presuming } a_{11}>a_{21} \text { not } a<1 \text {; NA }=\text { category not applicable. } \\
\text { b Form depends on the kernel } K(x) \text {. }\end{array}$} \\
\hline
\end{tabular}

Table 1: Primary results summary 


\begin{tabular}{|c|c|c|c|c|c|c|c|c|c|}
\hline Differences & $\mathrm{C}_{\mathrm{e} 2}<\mathrm{C}_{\mathrm{e} 1}$ & \multirow{2}{*}{\multicolumn{2}{|c|}{$\frac{h_{2}<h_{1}}{\text { Pop. } 2}$}} & \multirow{2}{*}{\multicolumn{3}{|c|}{$\frac{a_{21}<1<a_{12}}{\text { Pop. } 2}$}} & \multicolumn{3}{|c|}{$x_{1}{ }^{*}<x_{2}{ }^{*}$} \\
\hline$k_{1}>k_{2}$ & Pop. 2 & & & & & & Pop. 1 & & op. 2 \\
\hline \multirow{5}{*}{$\mathrm{C}_{\mathrm{e} 1}<\mathrm{C}_{\mathrm{e} 2}$} & \multirow{5}{*}{-} & & & Pop. 1 & Both & Pop. 2 & Pop. 1 & Pop. 2 & Pop. 1 \\
\hline & & \multirow{2}{*}{\multicolumn{2}{|c|}{ Pop. 2}} & & & & & & \\
\hline & & & & Pop. 1 & \multicolumn{2}{|c|}{ Both } & Pop. 1 & \multicolumn{2}{|c|}{ Pop. 2} \\
\hline & & \multicolumn{2}{|c|}{ Pop. 1} & & & & & & \\
\hline & & & & \multicolumn{3}{|c|}{ Pop. 1} & \multicolumn{3}{|c|}{ Pop. 1} \\
\hline \multirow{3}{*}{$h_{1}<h_{2}$} & \multirow{3}{*}{-} & & & & & & Pop. 1 & Pop. 2 & Pop. 1 \\
\hline & & \multirow{2}{*}{\multicolumn{2}{|c|}{ - }} & Pop. 1 & Both & Pop. 2 & \multicolumn{2}{|l|}{ Pop. 1} & Dop. 2 \\
\hline & & & & & & & \multicolumn{3}{|c|}{ Pop. 1} \\
\hline$a_{12}<1<a_{21}$ & - & \multicolumn{2}{|c|}{ - } & \multicolumn{3}{|c|}{-} & Pop. 1 & Both & Pop. 2 \\
\hline Resources & Low $\rightarrow$ High & \multicolumn{2}{|c|}{ Low $\rightarrow$ High } & \multicolumn{3}{|c|}{ Low $\rightarrow$ High } & \multicolumn{3}{|c|}{ Left $\rightarrow$ Right } \\
\hline
\end{tabular}

Figure 3: Competitive trade-offs. Each population is favored by one of the five competitive asymmetries. To read these results, take an outcome block-row for a given trade-off. The leftmost block characterizes the outcome at low resource levels, beginning at $R=C_{i e} h_{i}$. Blocks farther to the right correspond to alternative results that arise at sufficiently high resources (e.g., $R_{\mathrm{p} i}, R_{\mathrm{ri}}$ ). If the resource maximum is too low, these additional outcomes might not be fully realized. When the second population has an offset optimum, the reading of the blocks is in terms of position or index, $x$, and not explicitly resource, $R$. Where multiple outcome block-rows are listed for a given trade-off, the choice will be dependent on specific parameter values. Unlisted results are symmetric to those included in the table and have been omitted for clarity. Secondary variations in interference are not included.

range margins. Steady state populations overlap where $R>\max \left\{R_{\mathrm{p} 1}, R_{\mathrm{p} 2}\right\}$ (fig. $4 A, 4 B$ ). Alternatively, when interspecific competition is relatively stronger $\left(a>1, a_{i i}=\right.$ 1 ), condition (4) is inverted, indicating the devolution of distributions into a mosaic arrangement. Even populations that are metabolically less fit $\left(\mu_{i} / r_{i}\right)$ can persist through the deterrence of heightened intergroup aggression if they establish themselves early in high-resource regions (fig. $4 C$, 4D).

\section{Interference and Resource Collection}

Asymmetric competitive strengths, $a_{i j}$, and resource gathering times, $h_{i}$, are each individually sufficient to ensure long-term regional competitive exclusion, but these contrasts favor specific and distinct spatial patterns during the community's transient phase (fig. 2, cols. 2, 3; fig. 4). The arbitrary interchangeability of transient population densities that was characteristic of the two previous asymmetries in sensitivity and effective mortality is noticeably absent here.

Consider first interference dominance. Without loss of generality, assume that the first population has a disproportionately strong effect on the other $\left(a_{12}=1-\epsilon\right.$, $\left.a_{21}=1+\epsilon, a_{i i}=1\right)$ and that it has been introduced into an existing population (population 2) that is not necessarily at population equilibrium. During the transient phase, sympatric zones form around the central resource peak as the more aggressive population (population 1) initially relocates there before expanding outward (fig. 5). A subsequent expansion of exclusive territory then follows outward from the center (fig. $2 F$ ). Weaker individuals are literally marginalized as the perceived fitness benefit from a reduction in competitive pressures temporarily offsets the cost of reduced resources at the margins (figs. 2I, 4).

Under a simple asymmetry in interspecific interference, the weaker population is eventually regionally eliminated; however, additional variation in intraspecific interference can prevent this. Specifically, if $a_{22}=1$ and $a_{12}<1<$ $a_{21}$, then population 2 can both immigrate into and demographically persist in the territory of population 1 if $a_{21}<a_{11}$, that is, if the conspecific conflicts within an aggressive population (population 1) are more costly than heterospecific ones are for the meeker population (population 2; cf. this description with the classic hawk-dove scenario).

In contrast, transient ideal distributions under asymmetric resource gathering $\left(h_{1} \neq h_{2}\right)$ never exhibit local 

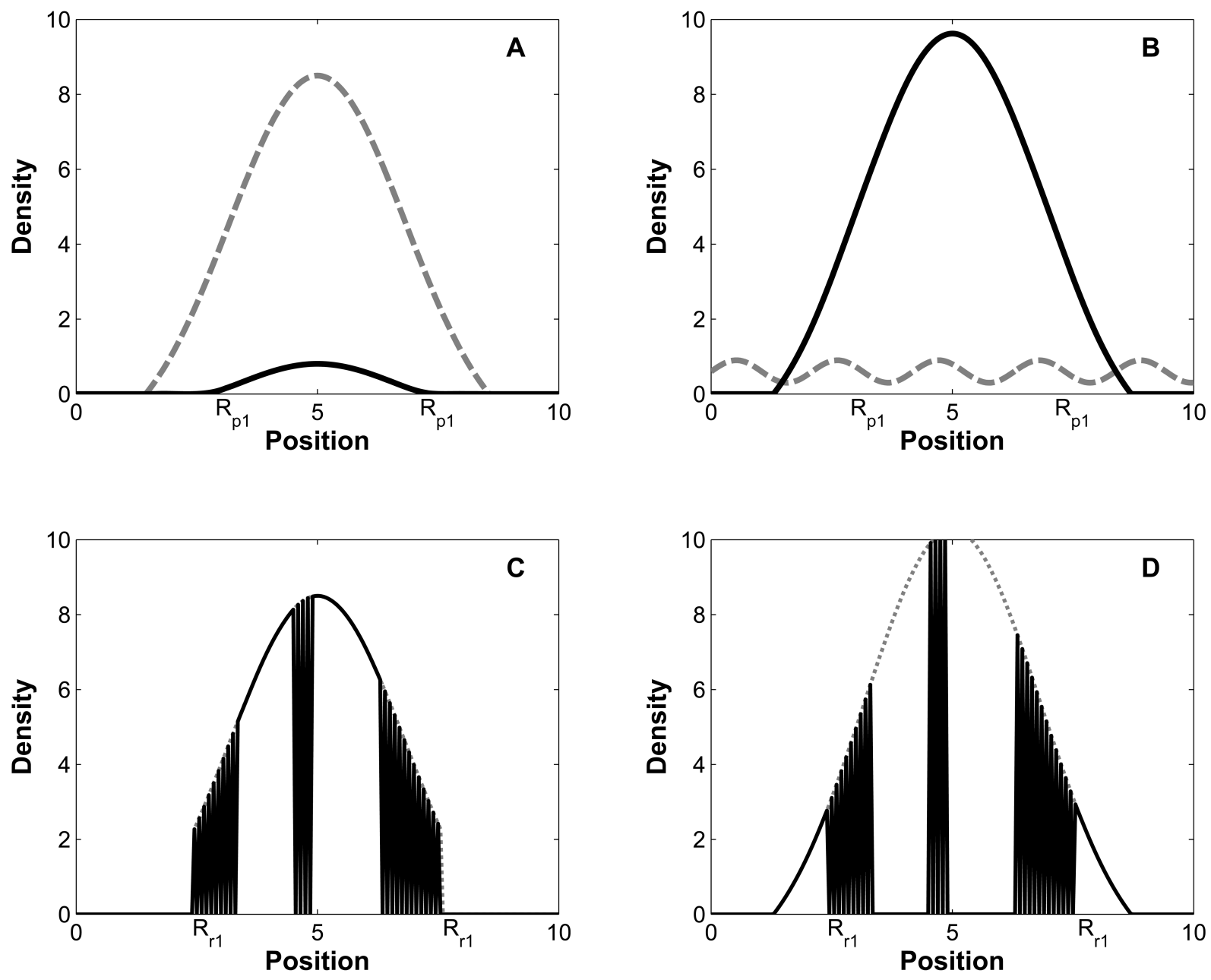

Figure 4: Persistence under secondary variation in interference. Two simulation runs were performed with interspecific pressures $a=0.8$ and $a=1.3$ for populations differing only in their per capita mortality $\left(k_{i}=0.2, h_{i}=1.5, a_{i i}=1, r_{i}=0.5\right)$. The resident population $\left(A\right.$, $C$; $\mu_{1}=$ $0.5)$ is initially at isolation equilibrium, $\tilde{u}_{1}$ (resource as in figs. 1,2$)$. A healthier invader population $\left(B, D ; \mu_{2}=0.425\right)$ is introduced at low nonuniform levels across the landscape $\left(u_{2}(x, 0)=0.6+0.3 \sin (3 x)\right.$ ). Curves shown: initial distributions (dashed; $\left.A, B\right)$, final distributions (solid), theoretical optimum distribution (dotted; $C, D)$. Lower interspecific interference $(a=0.8)$ : the resident population $(A)$ persists at low levels over a restricted range $(R(x)>5.1)$; the healthier population $(B)$ expands. Greater interspecific interference $(a=1.3)$ : fragmentation due to competitive instabilities allows persistence $(C)$ wherever $R(x)>3.64$; here, this results in three mosaic regions and two contiguous regions maintained by population 1. The fitter population $(D)$ maintains exclusivity to low-resource areas. Simulations were performed with Matlab software, using a simple forward difference-central difference approximation $(\Delta x=\Delta t=0.05)$.

co-occurrence. Sharp range limits form under fully normalized strength of competition (fig. 2E) because without differential interference, condition (4) is never satisfied, except in the trivial case of the boundary itself (fig. $2 B$ ). Furthermore, the central-peripheral ordering of the populations is reversed (fig. 5). The more efficient gatherers dominate the range margins and use resources that are not ordinarily available or desirable to the less adroit population before moving inward. As with effective mortality, however, differences between intra- and interspecific in- terference can mitigate the situation and provide some measure of transient sympatry or even persistence of the disadvantaged population in high-resource environments (table 1).

Of note, the development of ideal invasion patterns for efficient gatherers is critically dependent on the physical design of the environment. In the linear environment presumed in equation (2) (e.g., coastlines, rivers, or altitudinal or latitudinal gradients), the resident population constitutes a movement barrier to the invading population. In- 


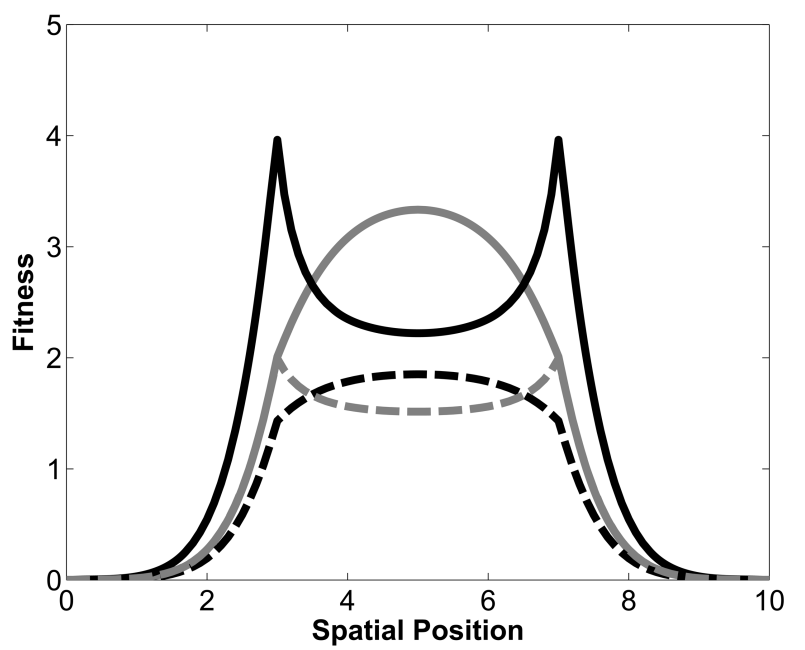

Figure 5: Invasion fitness landscape. Differences in territorial strength and resource collection produce opposing spatial tendencies. The invading population is small relative to the resident population, which is assumed to be at ideal equilibrium $\left(C_{1}=2, h_{1}=1\right)$ across $R(x)=$ $10 \exp \left[-0.4(x-5)^{2}\right]$. Strong invaders (gray line; $a_{21}=0.5$ ) favor an immediate concentration around peak resources. Superior collectors (black line; $h_{2}=0.5$ ) prefer marginal regions. Inferior populations reverse these patterns: weak fighters (dashed gray line) vacate to the margins, while slow collectors (dashed black line) gravitate to where resources are plentiful.

vaders will occupy only that half of the territory closest to their introduction (fig. $2 E$ ) up to the location of the resource maximum, after which that subpopulation stabilizes and an offshoot colony appears at the opposite end of the landscape (e.g., at the left fitness peak in fig. $2 H$ ). The original invader population constantly supports the satellite colony until the two subgroups finally merge, completing the elimination of the resident population. In the two-dimensional analog of equation (2), however, the existing population presents no equivalent barrier to movement between opposite ends of the range. Superior collectors circumvent their opponents, surrounding them in a ringlike distribution before the population boundaries collapse inward toward the resource peak.

\section{Optimal Locations on a Cline}

Consider now two populations distinguished not by a parameter in equation (2) but in the location of their tolerance range along an environmental cline. For simplicity, let us assume that this environmental variation manifests itself in the perception and utility of resources such that equations (1)-(4) use the resource utility curves $(i=$ $1,2)$ :

$$
R_{i}(x)=R_{\max } K\left(x-x_{i}^{*}\right),
$$

where $K$ is a nonnegative, single-humped distribution kernel with $K(0)=1$ and an outward limit of $K=0$ and $R_{\max }$ provides the appropriate scale. The kernels for the populations are centered at $x_{1}^{*}$ and $x_{2}^{*}$. Without some variation in interference strength, parapatry (or allopatry) is expected in both transient and steady state ideal distributions, but because each population has an effective refuge from competition centered about its respective optima, both populations regionally persist. The boundary between them is equidistant from those centers.

\section{Competitive Trade-Offs}

We have seen that variation in con- and heterospecific interference can prevent regional competitive exclusion produced by simple parametric asymmetries. Another possible solution is that competitors engage in some form of competitive trade-off, with each population advantaged in one fashion or other. Figure 3 contains the results of this secondary analysis when the simple advantages or differences discussed above are put into pairwise consideration.

Sensitivity has the least effect on the environmentally static model; populations better at resource collection, metabolizing resources, or imposing interspecific interference will regionally exclude more sensitive populations under equations (1) and (2). Effective mortality is a robust advantage to possess in that the population gains alternative benefits over competitors in both low- and high-resource environments and its inclusion in a trade-off creates an array of possible outcomes. Simple differences in collection times or clinal variations in resource utility impose strict parapatry for ideal distributions; however, clear dominance in interference strengths can override this rule and enforce sympatry at intermediate resource levels.

\section{Discussion}

This article considers the spatial implications of competitive asymmetries between two ideally motivated populations occupying the same ecological niche. Under traditional nonspatial dynamics, a population with an arbitrary performance or fitness advantage will be selected for at the expense of the other population in monotonic fashion, yet as shown here, an explicit spatial context broadens the possibilities for regional co-occurrence. By assessing local variations in resource and consumer levels, populations adopt distributions that best suit the particular asymmetries between them. Even when the ultimate outcome is regional exclusion, transitional patterns remain distinguishable. This article identifies five primary forms of asymmetry within the model, including four individual 
parametric contrasts and a shift in the populations' respective optimal locations along an environmental cline. A compounding factor of differences between interspecific interference and intraspecific interference is also examined. Summarized in table 1 and figure 3, this article's results were obtained through methods that are easily generalized to other models of competition or alternative community structures. These methods are also themselves important in that they further extend the concept of the IFD (Fretwell and Lucas 1969) to systems with both spatially continuous environments (Kshatriya and Cosner 2002; Cosner 2005; Rowell 2009) and multiple populations within a unified framework (Křivan and Sirot 2002; Křivan et al. 2008).

When multiple populations occupy the same relative niche, the natural inclinations to colonize high-resource areas first must adapt to the other population's presence. The populations interpret the available information differently during dispersal because of various performance asymmetries (e.g., fig. 5). The local IFD corresponding to each population's preferred response often appears unbalanced from the perspective of the other population, and this initiates a continual feedback of countermovements that persists until a mutually satisfying arrangement is reached (joint IFD). This phenomenon is wholly absent in traditional, well-mixed dynamics without spatial structure. Rowell and Servedio (2009) observed a similar phenomenon in the context of male mate selection where variation in males' valuation of different female phenotypes culminated in a strong mating assortment.

This article asks two questions of the populations' spatial distributions: is ideal sympatry ever possible, and do the populations have designated positions along the central-marginal axis of the resource distribution? The major results of this article can be characterized succinctly: awareness leads to a wider dispersal (sensitivity), health and vigor lead to displacement (effective mortality), the strong scatter the weak (interference), the skilled envelop the less adroit (collection time), and refuges promote survival (clinal variation). While some of these conclusions will strike the reader as intuitively obvious, it is important to emphasize that they follow directly from the limited assumption that individuals move in their own selfish interest. The collective group adopts a maneuvering approach that is tactically sound in the conventional sense of the term, without any group directive or agency assigned to the distribution.

Performance differences can be categorized as either nonobservable factors or observable interactions with the environment or with one another, based strictly on their inclusion in the operant search measure (e.g., $S_{i}$ in eq. [1]). Nonobservable asymmetries include internalized parameters such as sensitivity to information, metabolic ef- ficiency, and mortality rates. They are spatially neutral in organizing the border between distributions and in their assignment of populations to positions of high or low resources. The populations are blind to the differences between them and essentially regard one another as conspecifics (e.g., fig. $2 A$ ). This does not mean that no spatial differences are observed, but the effects of the asymmetry are indirect in nature, as there is no intrinsic tendency to integrate or segregate. Instead, populations with improved perception often achieve wider distributions by virtue of quicker colonization. Likewise, healthier individuals with lower mortality or greater metabolic efficiency depress the fitness of the disadvantaged population via overcrowding at the community level, displacing their competitors but not radically shifting their position.

In contrast, observable interactions with the environment are not spatially neutral. Both resource-gathering rates and clinal variations in optimal location present a binary assessment of the environment. A location is appropriate to either one population or the other but never simultaneously to both, and ideal sympatry cannot occur (fig. 2B, 2E). Where these modalities diverge is in the relative position of populations and the prospect for regional coexistence. Populations that quickly gather resources can leverage untapped marginal resources, enveloping their rivals clinging to the resource peak before boundaries crush inward, but populations that differ in their optima disengage and restrict themselves to more preferred areas of the environment.

Observable interactions within and between populations (e.g., interference) are also not spatially neutral, but with these asymmetries, the populations exhibit a tolerance for imperfect areas and can co-occur under some restrictions. Dominant populations concentrate around high-resource locations, ignoring the presence of weaker individuals marginalized to areas of poorer resources, and yet the two populations remain co-occurrent at intermediate resource levels so long as they are both regionally extant. Persistent sympatry is achievable with secondary variations within intraspecific competition (e.g., the hawk-dove scenario), and reduced interspecific pressures (e.g., partially overlapping resource needs or a "dear enemy" familiarity; see also Namba 1989) can mitigate the exclusion results of other asymmetries (table 1).

Animals often display more aggression over territorial resources toward nonkin (Reeve 1989), and elevated interspecific aggression is shown here also to support persistent regional co-occurrence (note the onset of spatial instability; see also Suárez 2004). For example, the Argentine ant, Linepithema humbile, demonstrates heightened aggression when it encounters ants from neighboring nest sites of other colonies (Thomas et al. 2007). The cost of competition is thought to be a clear public signal of de- 
terrence, with the risk of heightened aggression arresting potentially costly battles, in agreement with this model's exclusion threshold concept. A similar dynamic might be involved with hydrazoans (Hydractinia symbiolongicapus) competing for shell space on the hermit crab, Pagarus longicarpus (Thomas et al. 2007).

When there are multiple asymmetries between the populations (fig. 3), distributions follow a rough order of precedence. Variations in the strength of interference have first precedence in that they can impose sympatry over the strict parapatry expected from asymmetric interactions with the environment (e.g., collection speed, item handling, clinal variations). Spatially neutral distinctions cannot do so and default to the distributional rules of other asymmetries, for without differences in the strength coefficients to compensate for the divergence in group fitness levels, the sympatry condition (4) collapses to an easily falsified test of strict equality. Hence, parapatry is the default. Thus, a strongly interfering central population and a marginalized resource-gathering specialist could regionally coexist with sympatry at intermediate resource levels, but either strict parapatry or exclusion would result when those with lower effective mortality competed against superior collectors (fig. 2). Additionally, offsetting one's optimal location leads to regional coexistence, except in the most extreme trade-off circumstances.

The only parametric advantage that failed to persist in a trade-off was sensitivity. The key application of sensitivity is in colonization rates, but the environment of the model is static. Without regular disruption, the sensitivity advantage is employed only once at the community's onset and has no recurrent value among established populations. If environmental turnover or periodic fragmentation of the landscape could be incorporated into the model, however, sensitivity might prove a viable trade-off. For example, the common black rat, Rattus rattus, is a generally dominant invasive rodent that was once believed to have driven the Santiago rice rat, Nesoryzyomys swarthi, to extinction before the latter's rediscovery (Harris and MacDonald 2007). If the black rat marginalized the Santiago rice rat, $N$. swarthi would have been positioned to become isolated by seasonal or climatic changes, and greater sensitivity or speed of response could then have facilitated its persistence through discovery of impermanent remote refuges. Moreover, another competitor of the black rat, Mus musculus, is thought to be good at avoiding encounters with its larger rival-perhaps signaling another functional interpretation of the sensitivity in the model.

The theoretical literature has recorded similar results, but important qualifications distinguish the current findings from previous ones (e.g., phenomenological causes). Ito (1984) showed that a diffusive, weakly interfering population could persist in a peripheral distribution or even exclude the locally dominant population. Ito's model incorporated multiple size-linked asymmetries but lacked resource heterogeneity, and the observed distributions originated from a well-known phenomenological interaction between diffusion and absorbing ("zero Dirichlet") boundary conditions. The weaker population persisted via a functional trade-off in which it benefited from lower diffusion. By moving more conservatively, the smaller individuals made fewer lethal mistakes near the range limits than did larger ones. For Ali and Cosner (1995), the movement risk was generated by a piecewise elevation in the risk of predation away from the central area, but in both models, the viability of larger individuals was dependent on the size of "good" regions, with smaller individuals able to persist on smaller-quality sites and larger individuals appearing and then later dominating as these sites grew in size. Other diffusive competition models found coexistence when interspecific competition was relatively weaker than intraspecific competition (Namba 1989) and when there was heightened aggression between populations (Suárez 2004).

Křivan and Sirot's (2002) findings are more directly in line with those of this article because of the inclusion of a better response dynamic that drove individuals in pursuit of environments where they were fitter (see also Cressman and Krrivan 2006; Krrivan et al. 2008). They examined a two-patch model of two populations with patch-dependent growth rates and carrying capacities and employed a similar two-timescale approach to describe the model's behavior. Depending on the densities of the competing populations, the two populations could be spatially segregated, with the dominant species generally ignoring interspecific competition. Thus, it would remain in the best patch when at low density and invade lesser areas only when numerous. Also, very strong interspecific competition led to the existence of many possible distributions and the introduction of spatial instability to sympatric arrangements, mirroring the spatial instability of the armed-camp exclusion scenario identified in this article (see also Suárez 2004). Finally, the population cycling of Abrams et al.'s (2007) related model with patch-dependent trade-offs contrasts with this competition model in which dispersal and demographic equilibria coincide.

This model necessarily makes concessions of realism in the interest of tractability, including the availability and reliability of information, freedom of movement, and equitability in resource sharing between new and established residents. The first concern is mitigated by the restriction of information to the gradient level; however, the governing search measure need not accurately reflect the local dynamics (e.g., not being able to distinguish between group affiliations, mimicry, etc.), nor is the execution of movement without error (e.g., the inclusion of diffusion 
or error in the decision process). Additionally, much of the analysis has assumed that equations (1) and (2) formed a rapid model, where dispersal operated on a faster timescale than did local dynamics. Treating movement separately highlights the connection between distributions here and in the classical IFD, and the approach obviates concerns about the model's well posedness as the spatial instability due to elevated interspecific aggression is confinable; however, the separation of timescales might not be appropriate in all cases (e.g., at the continental invasion scale). Although a transient population might rarely obtain an exact IFD when timescales are comparable, the flow of densities still retains the ghost of that objective.

A related consideration is that this article's conditions are necessary but insufficient to guarantee the presence of a given ideally motivated population in a designated location because of competitive fragmentation of the landscape. There must also have existed some initial historical distribution and opportune path of migration that gave the population access to the appropriate subregions, without which the indicated area remains empty or occupied by the other population (e.g., fig. $2 \mathrm{E}, 2 \mathrm{H}$; cf. distributions across multiple resource peaks in Rowell 2009). An important biological corollary serves as a warning for conservation efforts that direct observations of current population densities could fail to identify areas of greatest vulnerability to invasion because they are at some distance removed from the current interface between species' ranges.

This article advances a relatively recent approach to understand the spatial configuration of communities, but there remains a need for further development of the community-level theory and the observational and experimental testing of the qualitative predictions for the spatial patterns of competing populations. It would be useful to identify at what scale the local IFD is valid compared to historic population movements and which taxa are more inclined to be ideally motivated. Moreover, invasion patterns should recapitulate the natural distribution tendencies, with invaders adopting advantage-specific routes into the territory of an established population. This could be tied to current trends in geographic information system mapping of habitat suitability. If the theoretical conclusions of this article are supportable, it could provide a basis for improved management decisions. One interesting test case is the introduction of the American red-eared slider, Trachemys scripta elegans, to the Mediterranean region, where it has been outcompeting local turtles such as the Spanish terrapin, Mauremys leprosa (Polo-Cavia et al. 2009). A number of different mechanistic issues are involved in the problem, as sliders have multiple advantages over their native counterparts (bigger, more diverse diets, greater fecundity, and greater environmental toler- ance) but are less sensitive to the semiochemicals that both species release into the water to mark territory and navigate.

At the theoretical level, the analytic and graphical methods described here are robust and can be generalized to other spatially explicit models, with fitness or search functions defined in the broadest sense to include reproductive opportunities, resource availability, shelter from the elements and predators, and so on. Future theoretical developments include the calculation of ideal invasion speeds, the interaction of predator avoidance and prey searching, mismatches in the search measure and the corresponding dynamical feature (source-sink dynamics, dishonest signaling in territorial aggression and defense), multiple competitors (rock-paper-scissors), and genetically explicit problems such as the effect that ideal motivation has on hybridization or polymorphism maintenance or its role in speciation by spatially enforcing assortative mating. Finally, other biologically relevant modeling developments include shifting environmental conditions and instances where movement is phenotypically mediated, for example, the sex-mediated spatial positioning among ungulates during much of the nonbreeding period (Main 2008) or the size and resource-dependent movement speed of aquatic snails (Snider and Gilliam 2008).

\section{Acknowledgments}

This postdoctoral research was partially supported by National Institutes of Health grant GM-56693 awarded to S. Gavrilets of the University of Tennessee and later by National Science Foundation grant DEB-0614166 awarded to M. R. Servedio of the University of North Carolina at Chapel Hill. Significant work was performed at both institutions. I would like to thank J. Adamson, A. Chunco, S. Dhole, A. Frame, M. Servedio, and two reviewers for comments on the manuscript.

\section{Literature Cited}

Abrams, P. A., R. Cressman, and V. Krrivan. 2007. The role of behavioral dynamics in determining the patch distributions of interacting species. American Naturalist 169:505-518.

Ali, S. W., and C. Cosner. 1995. Models for the effects of individual size and spatial scale on competition between species in heterogeneous environments. Mathematical Biosciences 127:45-76.

Armsworth, P. R., and J. E. Roughgarden. 2005. The impact of directed versus random movement on population dynamics and biodiversity patterns. American Naturalist 165:449-465.

Berg, H. C. 1993. Random walks in biology. Princeton University Press, Princeton, NJ.

Case, T. J., R. D. Holt, M. A. McPeek, and T. H. Keitt. 2005. The community context of species' borders, ecological and evolutionary perspectives. Oikos 108:28-46. 
Connell, J. H. 1980. Diversity and the coevolution of competitors, or the ghost of competitors past. Oikos 35:131-138.

Cosner, C. 2005. A dynamic model for the ideal free distribution as a partial differential equation. Theoretical Population Biology 67: 101-108.

Cressman, R., and V. Krrivan. 2006. Migration dynamics for the ideal free distribution. American Naturalist 168:384-397.

Edelaar, P., A. M. Siepielski, and J. Clobert. 2008. Matching habitat choice causes directed gene flow: a neglected dimension in evolution and ecology. Evolution 62:2462-2472.

Fretwell, S. D., and H. L. Lucas. 1969. On territorial behavior and other factors influencing habitat distribution in birds. Acta Biotheoretica 19:16-36.

Fryxell, J. M., J. F. Wilmshurst, and A. R. E. Sinclair. 2004. Predictive models of movement by Serengeti grazers. Ecology 85:2429-2435.

Gaston, K. J. 2003. The structure and dynamics of geographic ranges. Oxford University Press, Oxford.

Goldberg, E. E., and R. Lande. 2007. Species' borders and dispersal barriers. American Naturalist 170:297-304.

Grindrod, P. 1988. Models of individual aggregation or clustering in single and multi-species communities. Journal of Mathematical Biology 26:651-660.

Hancock, P. A., and E. J. Milner-Gulland. 2006. Optimal movement strategies for social foragers in unpredictable environments. Ecology 87:2094-2102.

Harris, D., and D. W. MacDonald. 2007. Interference competition between introduced black rats and endemic Galápagos rice rats. Ecology 88:2330-2344.

Hofbauer, J., and K. Sigmund. 1998. Evolutionary games and population dynamics. Cambridge University Press, Cambridge.

Holt, R. D., and T. H. Keitt. 2005. Species' borders: a unifying theme in ecology. Oikos 108:3-6.

Ito, M. 1984. Global aspect of steady states for competitive-diffusion systems with homogeneous Dirichlet boundary conditions. Physica D 14:1-28.

Kareiva, P., and G. Odell. 1987. Swarms of predators exhibit preytaxis if individual predators use area-restricted search. American Naturalist 130:233-270.

Kirkpatrick, M., and N. H. Barton. 1997. Evolution of a species' range. American Naturalist 150:1-23.

Krrivan, V., and E. Sirot. 2002. Habitat selection by two competing species in a two-habitat environment. American Naturalist 160: 214-234.

Křivan, V., R. Cressman, and C. Schneider. 2008. The ideal free distribution: a review and synthesis of the game-theoretic perspective. Theoretical Population Biology 73:403-425.

Kshatriya, M., and C. Cosner. 2002. A continuum formulation for the ideal free distribution and its implications for population dynamics. Theoretical Population Biology 61:277-284.

Lima, S. L., and P. A. Zollner. 1996. Towards a behavioral ecology of ecological landscapes. Trends in Ecology \& Evolution 11:131135.

López-Gómez, J., and M. Molina-Meyer. 2006. Competitive exclusion principle versus biodiversity through competitive segregation and further adaptation to spatial heterogeneities. Theoretical Population Biology 69:94-109.

Main, M. B. 2008. Reconciling competing ecological explanations for sexual segregation in ungulates. Ecology 89:693-704.

Mari, L., R. Casagrandi, M. Gatto, T. Avgar, and R. Nathan. 2008. Movement strategies of seed predators as determinants of plant recruitment patterns. American Naturalist 172:694-711.

Moorcroft, P. R., and M. A. Lewis. 2006. Mechanistic home range analysis. Princeton University Press, Princeton, NJ.

Namba, T. 1989. Competition for space in a heterogeneous environment. Journal of Mathematical Biology 27:1-16.

Peterson, A. T. 2003. Predicting the geography of species' invasions via ecological niche modeling. Quarterly Review of Biology 78: 419-433.

Podos, J., and P. S. Warren. 2007. The evolution of geographic variation in birdsong. Advances in the Study of Behavior 37:403-458.

Polo-Cavia, N., P. López, and J. Martn. 2009. Interspecific differences in chemosensory responses of freshwater turtles: consequences for competition between native and invasive species. Biological Invasions 11:431-440.

Ravigné, V., I. Olivieri, and U. Dieckmann. 2004. Implications of habitat choice for protected polymorphisms. Evolutionary Ecology Research 6:125-145.

Reeve, H. K. 1989. The evolution of conspecific acceptance thresholds. American Naturalist 133:407-435.

Rosenzweig, M. L. 1991. Habitat selection and population interactions: the search for mechanism. American Naturalist 137(suppl.): S5-S28.

Rowell, J. T. 2009. The limitation of species range: a consequence of searching along resource gradients. Theoretical Population Biology 75:216-227.

Rowell, J. T., and M. R. Servedio. 2009. Gentlemen prefer blondes: the evolution of mate preference among strategically allocated males. American Naturalist 173:12-25.

Schoener, T. W. 1983. Field experiments on interspecific competition. American Naturalist 122:240-285.

Shigesada, N., K. Kawasaki, and E. Teramoto. 1979. Spatial segregation of interacting species. Journal Theoretical Biology 79:8399.

Snider, S. B., and J. F. Gilliam. 2008. Movement ecology: size-specific behavioral response of an invasive snail to food availability. Ecology 89:1961-1971.

Suárez, A. 2004. Nonnegative solutions for a heterogeneous degenerate competition model. Australian and New Zealand Industrial and Applied Mathematics Journal 46:273-297.

Thomas, M. L., C. M. Payne-Makrisâ, A. V. Suarez, N. D. Tsutsui, and D. A. Holway. 2007. Contact between supercolonies elevates aggression in Argentine ants. Insectes Sociaux 54:225-233.

Vergassola, M., E. Villermaux, and B. I. Shraiman. 2007. "Infotaxis" as a strategy for searching without gradients. Nature 445:406-409.

Wilson, E. O. 1980. Sociobiology. Harvard University Press, Cambridge, MA.

Associate Editor: Vlastimil Křivan Editor: Donald L. DeAngelis 